\title{
RELATIONSHIPS BETWEEN CELL MIGRATION, ADHESION, APOPTOSIS AND GENE EXPRESSION IN FREE AND ATTACHED PERITONEAL CELLS IN TURBOT AFTER ADMINISTRATION OF VACCINES CONTAINING $P$. dicentrarchi ANTIGEN AND DIFFERENT ADJUVANTS
}

\author{
Francisco Fontenla ${ }^{1}$, José M. Leiro ${ }^{2}$, Manuel Noia ${ }^{1}$, Iria Folgueira ${ }^{1}$, Verónica Blanco- \\ Abad ${ }^{1}$, Antonio Gómez-Tato ${ }^{3}$, Belén G. Pardo ${ }^{4}$, Paulino Martínez ${ }^{4}$, Jesús Lamas ${ }^{1 *}$ \\ ${ }^{I}$ Department of Cell Biology and Ecology, ${ }^{2}$ Laboratory of Parasitology, Department of \\ Microbiology and Parasitology, ${ }^{3}$ Department of Geometry and Topology and ${ }^{4}$ Department of \\ Genetics, University of Santiago de Compostela, Spain
}

\begin{abstract}
Intraperitoneal (i.p.) injection is the most effective method of vaccinating fish in terms of antibody production and the degree of protection induced, and most current vaccines are administered in fish via this route. Vaccines are known to generate an inflammatory reaction in the peritoneal cavity, which is mainly caused by the adjuvant. Vaccination induces bidirectional movement of cells in and out of the peritoneal cavity where free cells and cells attached to the mesothelium and forming cell-vaccine masses can be observed. However, many aspects of the cellular immune response generated in the peritoneal cavity, such as the processes that provoke those cell responses, remain unknown. The present study analyses the cell traffic, adhesion, apoptosis and changes in gene expression in free and attached peritoneal cells after administration of vaccines containing $P$. dicentrarchi antigen and different types of adjuvants. Turbot were injected with PBS, PBS plus $P$. dicentrarchi membrane antigen, microspheres of chitosan-PVMMA containing membrane antigen, or with a mixture of antigen and one the following adjuvants: Montanide Isa 763 A, aluminium hydroxide gel or Matrix-Q. A control group also included non injected fish. Fish were sampled at several times after vaccination. The peritoneal cavity was washed with $5 \mathrm{~mL}$ of cold PBS to obtain the free peritoneal cells. Cells forming cell-vaccine masses and cells attached to the peritoneal mesothelium were also obtained and disaggregated by treatment with trypsin and EDTA. Cells were analysed by light microscopy, immunohistochemistry and flow cytometry. Gene expression was determined in free peritoneal cells by using a microarray rich in genes related to the immune response and to the cell cytoskeleton. In addition, gene expression was analysed by real-time PCR analysis of attached peritoneal cells and of spleen and kidney cells. The results indicate intense cell traffic to and from the peritoneal cavity, including free cells, cells attached to the mesothelium and others migrating to lymphoid organs. Gene expression analysis revealed intense upregulation of genes associated with cytoskeleton, cell adhesion and cell surface receptors. Apoptosis was observed from day one, with upregulation of genes involved in apoptosis and cell death. However, lymphocytes were scarce, and downregulation of genes related to lymphocyte activity and lymphocyte chemotaxis was observed. Finally, we compared the responses generated by the different adjuvants.
\end{abstract}


KEYWORDS: Turbot, vaccination, immune response, peritoneal cells, gene expression, Philasterides dicentrarchi

*Corresponding author. Tel: +34881816951

e-mail: jesus.lamas@usc.es 